\title{
LIVIA VISSER-FUCHS, History as Pastime. Jean de Wavrin and His Collection of Chronicles of England
}

\author{
Maria Colombo Timelli
}

\section{(2) OpenEdition}

\section{Journals}

Édition électronique

URL : https://journals.openedition.org/studifrancesi/22367

DOI : $10.4000 /$ studifrancesi.22367

ISSN : 2427-5856

Éditeur

Rosenberg \& Sellier

Édition imprimée

Date de publication : 1 avril 2020

Pagination : 154-155

ISSN : 0039-2944

Référence électronique

Maria Colombo Timelli, « LIVIA visser-Fuchs, History as Pastime. Jean de Wavrin and His Collection of Chronicles of England », Studi Francesi [En ligne], 190 (LXIV | I) | 2020, mis en ligne le 01 avril 2020, consulté le 03 août 2021. URL : http://journals.openedition.org/studifrancesi/22367 ; DOI : https:// doi.org/10.4000/studifrancesi.22367

Ce document a été généré automatiquement le 3 août 2021

\section{(c) (i) (9)}

Studi Francesi è distribuita con Licenza Creative Commons Attribuzione - Non commerciale - Non opere derivate 4.0 Internazionale. 


\title{
LIVIA VISSER-FUCHS, History as Pastime. Jean de Wavrin and His Collection of Chronicles of England
}

\author{
Maria Colombo Timelli
}

\section{RÉFÉRENCE}

LIVIA VISSER-FUCHS, History as Pastime. Jean de Wavrin and His Collection of Chronicles of England, Donington, Shaun Tyas, 2018, 682 pp. +13 planches en couleur.

1 Personnage bien connu par les historiens du livre manuscrit, Jean de Wavrin fait ici l'objet pour la première fois d'une monographie qui, tout en mettant l'accent sur son activité d'historien amateur et sur sa seule production, le Recueil des Croniques d'Engleterre, permet d'en appréhender la personalité dans toute sa complexité. Soldat professionnel, auteur de chroniques, collectionneur de manuscrits, Jean de Wavrin occupe en effet une place particulière sur une scène «triangulaire», entre France, Angleterre et Bourgogne, comme le rappelle l'Introduction (pp. 1-11).

2 Sa biographie fait l'objet du premier chapitre (Life, Family and Friends, pp. 12-97): né entre octobre 1399 et 1400, fils illégitime de Robert VII de Wavrin, grand aristocrate picard, Jean participa à la bataille d'Azincourt dans l'armée française; sa carrière militaire s'interrompit cependant en 1436 déjà. Légitimé en 1437, il épousa une veuve appartenant à une famille de drapiers, pour devenir ensuite bourgeois de Lille et, vers 1441, seigneur de Forestel, puis capitaine de Lillers. Entré au service de Philippe le Bon, il voyagea en Italie, à Venise et à Rome, en tant que diplomate et ambassadeur dans le cadre du projet de Croisade cher au Duc (1463-1467). C'est au cours de la dernière période de sa vie, sous Charles le Téméraire, qu'il rédigea son recueil de Chroniques, à partir de sources déjà existantes et parfois intégrées telles quelles à son œuvre. L.V.-F. essaie aussi de reconstituer le réseau de ses proches, son neveu Waleran, ainsi que les amis dont il partageait les goûts et les intérêts, autant de noms bien connus dans le 
milieu des ducs de Bourgogne: Jean de Crequy, Hugues et Guillebert de Lannoy, et encore Philippe de Lalaing, Hubert le Prévost, Jacques du Clercq, Jean Lefèvre de SaintRemy, sans oublier Philippe le Bon lui-même, dont la collection comprenait au moins cinq manuscrits appartenus à Jean de Wavrin sans qu'il soit aujourd'hui possible d'en établir sûrement l'histoire.

Le chapitre 2 (Wavrin's Books, pp. 98-235) aborde au moins quatre questions; tout d'abord, l'attribution à Jean de Wavrin lui-même d'un certain nombre de romans en prose: Florimont, Comte d'Artois, Seigneurs de Gavre, Girart de Nevers, Gillion de Trazegnies, Chastelain de Coucy, Gilles de Chin, Jacques de Lalaing; rien n'est cependant moins sûr, les seules certitudes concernant l'illustration de ces manuscrits - de la main du «Maître de Jean de Wavrin» - et la présence de cinq de ces copies dans la «librairie» de Jean (Comte d'Artois, Seigneurs de Gavre, Gillion de Trazegnies, Gilles de Chin, Jacques de Lalaing). Deuxième question, déjà bien balayée, la composition de sa bibliothèque; l'analyse peut s'appuyer sur des éléments plus solides: la présence de marques de propriété et/ou la mention des titres dans les inventaires, entre autres celui de Marguerite d'Autriche. L.V.-F. répartit les livres sous les rubriques «Fiction», qui comprend des titres comme Olivier de Castille et Artus d'Algarbe, Paris et Vienne, Apollonius de Tyr, Buscalus, Jean d'Avennes, Jean de Saintré et d'autres encore, «Non-fiction» (religion, morale, technique militaire, histoire, médecine), et ajoute des remarques sur d'autres ouvrages qui, sans lien avéré avec Jean de Wavrin, seraient proches de ses intérêts (Blancandin ou Cligés en prose, par exemple). Troisième sujet: le «Maître de Wavrin», miniaturiste bien connu dont l'originalité - peinture sur papier à l'aquarelle, figures presque caricaturales - a fait couler beaucoup d'encre et a fait l'objet de la thèse fondamentale de Pascal Schandel (1997, malheureusement inédite); L.V.-F. se concentre en particulier sur les miniatures de présentation et sur le passage de certains manuscrits de la collection Wavrin à la «librairie» de Philippe le Bon. Quatrième et dernière question: la présence des Anglais dans les livres possédés par Jean de Wavrin, une présence somme toute discrète et qui ne révèle aucun signe ou connaissance particulière de l'Angleterre. La conclusion de ce long chapitre ne surprend pas: Jean de Wavrin a réuni une collection dont l'ampleur ne fait pas de doute - même en l'absence de tout inventaire - ni d'ailleurs l'homogénéité, ce qui prouve, au-delà d'un goût certain pour les livres, un réseau de connaissances dans les milieux des copistes et enlumineurs lillois de la seconde moitié $\mathrm{du} \mathrm{Xv}^{\mathrm{e}}$ siècle.

4 Les chapitres restants portent tous sur les Croniques d'Engleterre. L.V.-F. offre d'abord une vue d'ensemble de l'œuvre: dédicace à Waleran, dates de rédaction ( $c a$ 1455-1473/4), structure (titre, organisation en quatre, puis en six livres, ajout sur l'expédition de Waleran contre les Sarrazins), modalités de composition (par compilation de sources diverses), propos de l'auteur (remplir un vide, en composant une histoire complète de l'Angleterre), copies conservées (liste en Appendice E; une attention particulière est apportée ici aux manuscrits ayant appartenu à Louis de Bruges, Engelbert de Nassau, Edouard IV); l'analyse du programme iconographique reste à faire, alors que l'identité des illustrateurs ne fait pas l'accord des critiques. Toujours est-il que Jean de Wavrin semble avoir atteint le lectorat qu'il visait, à savoir les grands aristocrates du milieu bourguignon ainsi que ses proches (chapitre 3, The "Recueil des Croniques d'Engleterre", pp. 236-315).

5 Il s'agit ensuite de détecter les sources utilisées par Jean de Wavrin, déjà reconnues par la critique, et surtout de vérifier ses techniques de réécriture et d'inclusion: si une telle 
recherche est relativement aisée pour la tranche allant de la fondation d'Albion à ca 1400 , elle se fait plus compliquée pour le $\mathrm{xv}^{\mathrm{e}}$ siècle. En adoptant une présentation chronologique, L.V.-F. discute d'abord les sources possibles pour l'histoire d'Albina, pour passer ensuite au traitement réservé à l'Historia regum Britanniae de Geoffroi de Monmouth, au Brut et à la Grande Chronique de Normandie, aux Chroniques de Froissart enfin (chapitre 4, Sources and Method, Albina to c. 1400: One Romance and Four Chronicles, pp. 316-343). Le chapitre suivant porte sur les dernières décennies, pour lequelles Jean de Wavrin disposait de plusieurs sources différentes qu'il n'hésita pas à exploiter en même temps: Enguerrand de Monstrelet, Jean Lefèvre de Saint-Remy, et bien d'autres chroniqueurs (Sources and Method, c. 1400-1471/72. Part I. Contemporary Chronicles, pp. 344-380); à ceux-ci s'ajoutent encore des sources diverses et moins étendues, comptes-rendus de tous genres relatant tant des épisodes de guerre que des cérémonies religieuses ou civiles, voire des désastres naturels, et encore des lettres, parfois signées, dont il est encore plus délicat de repérer la présence à l'intérieur des Croniques d'Engleterre, à plus forte raison lorsque les documents originaux ne semblent pas conservés (chapitre 6, Sources and Method, c. 1400-1471/72. Part II. Newsletters, pp. 381-442).

Dans un dernier chapitre L.V.-F. s'interroge sur les deux faces de Jean de Wavrin, à la fois soldat et homme de cour, homme de guerre et aristocrate bourguignon (chapitre 7, Wavrin himself, pp. 443-508). Pour relater les batailles, qui forment le noyau de ses Chroniques, Wavrin utilisa autant que possible des sources existantes, sans exclure, le cas échéant, ses propres souvenirs et expériences; néanmoins, on peut toujours s'interroger sur la valeur objective de ses portraits et opinions, au vu entre autres des similarités entre ses récits et ceux qui se lisent dans la littérature romanesque de l'époque. Quant à son rôle dans les milieux cultivés de la cour, au-delà de sa compilation des Chroniques, c'est en tant que collectionneur de manuscrits, commanditaire de textes et d'images, et surtout proche de Philippe le Bon - avec lequel les échanges dans ce domaine furent nombreux et fréquents - que Jean de Wavrin joua un rôle important dans la cour bourguignonne de son temps.

$7 \quad$ Le bilan de ce gros ouvrage est tiré en quelques pages (By way of Conclusion: the Value of the "Recueil", pp. 509-514), où L.-V.F. synthétise la méthode que Wavrin suivit pour compiler son œuvre, qui nous révèle entre autres les sources dont il disposait; par ailleurs le caractère disparate de celles-ci, ainsi que l'intervention des copistes (aucun autographe des Croniques ne nous est parvenu), peuvent expliquer certaines imprécisions dans la chronologie des événements ou dans leur géographie. Au total, on serait tenté de dire que l'intérêt de cette histoire de l'Angleterre réside en grande partie dans le système adopté par son auteur, capable de collecter des textes et des documents divers afin d'offrir à ses lecteurs premiers, les grands aristocrates bourguignons, une histoire qui soit à la fois utile et agréable.

8 Parmi les nombreux appendices et annexes, signalons la liste des manuscrits ayant appartenu à Jean de Wavrin, ou associés à son nom (Appendice B, pp. 518-542: 53 entrées, avec présentation matérielle et commentaire), la liste des manuscrits du Recueil à laquelle on a déjà fait allusion (Appendice E, pp. 555-572), la transcription de quelques prologues de romans associés à Jean de Wavrin (Appendice F, pp. 573-583, avec relevé de quelques formules et clichés récurrents). La Bibliographie, déjà très vaste (pp. 601-647), peut être complétée grâce à la parution récente du volume L'art du récit à la cour de Bourgogne. L'activité de Jean de Wavrin et de son atelier, Paris, Champion, 2018 (actes d'un colloque de 2013); dans une perspective littéraire mériteraient aussi 
d'être ajoutés les articles de: Isabelle Arseneau 2015, Les mises en prose de l'atelier du Maître de Wavrin: pistes et réflexions, in Les Centres de production des manuscrits vernaculaires au Moyen Âge, Paris, Classiques Garnier, 2015 pp. 201-215; Annie Combes, L'athanor de la prose (l'atelier de Jean de Wavrin), in «Le Moyen Français» 76-77, 2016, pp. 39-64; Rosalind Brown-Grant, Visualizing Justice in Burgundian Prose Romance: the "Roman de Gérard de Nevers Illuminated by the Wavrin Master and Loyset Liédet", in "Gesta" 57/1, 2018, pp. 69-96. 